PFC/JA-91-20

\title{
Parametric Studies of Free Electron Laser Nonlinear Ponderomotive Trapping Buckets
}

\author{
J. Fajans, R. Chu, J.S. Wurtele
}

\author{
June, 1991 \\ Plasma Fusion Center \\ Massachusetts Institute of Technology \\ Cambridge, MA 02139 USA
}

This work was supported by the National Science Foundation, the Office of Naval Research, the U.S. Department of Energy, Division of Nuclear and High Energy Physics, and by internal U.C. Berkeley funds. Reproduction, translation, publication, use and disposal, in whole of part, by or for the United States government is permitted.

Submitted for publication in IEEE Journal on Quantum Electronics, Special Issue on Free Electron Lasers. 


\title{
Parametric Studies of Free Electron Laser Nonlinear Ponderomotive Trapping Buckets
}

\author{
J. Fajans \\ Department of Physics \\ University of California, Berkeley \\ Berkeley, California 94720 \\ R. Chu and J.S. Wurtele \\ Dept. of Physics and Plasma Fusion Center \\ Massachusetts Institute of Technology \\ Cambridge, MA 02139
}

June 27, 1991

\begin{abstract}
Experimental and computational studies of free electron lasers operating at high input powers have been undertaken. These studies constitute a novel method of investigating saturation and trapping effects in free electron lasers.
\end{abstract}

A crucial issue in the practical application of free electron lasers (FELs) is the maximization of output efficiency. Since high efficiency operation inevitably occurs in the nonlinear regime, understanding FEL nonlinear behavior is very important. However, although FEL linear behavior is now well explored and understood, there have been relatively few experimental studies of FEL nonlinear behavior. Most saturated FEL experiments have run as oscillators $[1,2,3,4,5]$, where, because of the lack of precise control 
of the input conditions, it is difficult to carefully study the FEL behavior. A few experiments have run as amplifiers $[6,7,8,9,10]$, the most detailed studies to date having been the elegant tapering experiments at LLNL[7]. In this Letter we introduce a new nonlinear phenomenon directly related to the fundamental FEL nonlinear saturation mechanism[11].

We find both in experiments and simulations that when the input radiation power is increased to levels comparable to output power, the energy that maximizes the FEL gain shifts upwards. This effect is directly related to the height of the FEL ponderomotive trapping bucket[11]. By parametrically surveying the extent of this energy shift we observe nonlinear trapping bucket phenomenon. In some surveys the experimental results are well fitted by computer simulations, however for other surveys the experimental results are not in close agreement with simulations.

While this high input radiation power regime at first appears to be slightly artificial, it is in fact precisely the regime in which all saturated low gain, multipass oscillators operate. In such devices the output power generally builds from low noise levels by multiple reflections. Initially the device is in the linear regime, but it eventually leaves this regime for the high input power regime described in this paper. Since the operation is significantly different in the high input power regime, we predict that optimal behavior may require a shift in the system parameters once saturation is achieved.

It is easy to understand the origin of the energy shifts by considering the FEL longitudinal position and energy $(\Psi, \gamma)$ phase space (Fig. 1). In this phase space electrons with energy, $\gamma$, greater (less) than the resonant energy freestream forward (backward) in phase, $\Psi$. As usual, the resonant energy is defined to be the energy such that $\omega=\left(k+k_{w}\right) v_{z}$, where $\omega$ is the radiation frequency, $k$ is the radiation output wavenumber, $k_{w}=2 \pi / l$ is the wiggler wavenumber, and $v_{x}$ is the axial beam velocity. The velocity $v_{z}$ that satisfies this condition defines the resonant beam energy, $\gamma_{\text {res }}=1 /\left(1-v_{z}^{2} / c^{2}\right)^{1 / 2}$. The FEL interaction causes electrons with $\Psi>0(\Psi<0)$ to move down (up) in energy.

Our FEL operates in the Raman (collective) regime, and we will restrict the remainder of our discussion to this regime. Similar arguments hold for the other operating regimes (i.e. strong pump or temperature limited). In the linear regime the energy that maximizes the gain is shifted upwards from the resonant energy by an amount proportional to the beam plasma frequency. Likewise, the energy that results in maximum absorption of energy from 
the radiation to the beam is lowered by an equal amount. Thus the actual beam/radiation resonances occur at velocities shifted by the beam plasma frequency[12];

$$
\omega=\left(k+k_{w}\right) v_{z} \pm p_{1} \Phi^{1 / 2} \omega_{p} / \gamma_{\|} \gamma^{1 / 2}
$$

where $\omega_{p}$ is the nonrelativistic plasma frequency, $\gamma_{\|}$is the relativistic contraction resulting from the beam axial velocity only, $p_{1}$ is the reduction to the plasma frequency resulting from the finite beam radius and waveguide walls, and $\Phi \approx 1$ is a factor[13], not germaine to the phenomena under investigation in this paper, resulting from the interaction between the wiggler and axial magnetic fields. Using Eq. 1, we can determine the beam energy $\gamma_{\text {plin }}$ which results in the peak linear gain-i.e. the peak gain parameters are

$$
\begin{aligned}
& v_{\text {plin }}=\frac{\omega+p_{1} \Phi^{1 / 2} \omega_{p} / \gamma_{\|} \gamma^{1 / 2}}{k+k_{w}} \\
& \gamma_{\text {plin }}=1 /\left(1-v_{\mathrm{plin}}^{2} / c^{2}\right)^{1 / 2}
\end{aligned}
$$

This energy corresponds to exciting a slow space-charge wave on the beam. While a beam energy of exactly $\gamma_{\text {plin }}$ will result in the maximum gain, beams with energy in a small region around $\gamma_{\mathrm{plin}}$ will also result in gain. Typically, however, this region is significantly smaller than the energy difference between $\gamma_{\mathrm{plin}}$ and $\gamma_{\mathrm{res}}$. We can also define an equivalent $\gamma_{\mathrm{dlin}}$ corresponding to exciting the RF absorbing fast space-charge wave. The gain as a function of energy is shown in the detuning curve of Fig. 2.

At high RF powers, electrons inside an elliptically shaped separatrix centered around $\left(\gamma=\gamma_{\mathrm{res}}, \Psi=0\right)$ are trapped and execute circular orbits. These electrons are said to be in the FEL ponderomotive trapping "bucket". The height of the RF trapping bucket scales as[14]

$$
\Delta \gamma_{\text {trap }} \propto P^{1 / 4} B_{w}^{1 / 2}
$$

where $P$ is the RF power, and $B_{w}$ is the wiggler field[15]. So long as the input power is sufficiently low, the FEL will operate in the linear regime. Specifically, linear operation in Raman regime requires that the trapping energy $\gamma_{\text {trap }}=\gamma_{\text {res }}+\Delta \gamma_{\text {trap }}$ be significantly less than the linear gain energy $\gamma_{\text {plin }}$.

When the FEL input power is increased to the point that the trapping energy $\gamma_{\text {trap }}$ is comparable to the linear gain energy $\gamma_{\text {plin }}$, the beam electron 
dynamics will be influenced by the trapping forces. In the very high input power limit, the dynamics will be primarily determined by the trapping forces. The peak linear gain energy, $\gamma_{\text {plin }}$, will be less relevant. In fact, to take advantage of the extreme height of the trapping bucket, the energy that gives maximum gain will likely increase with $\gamma_{\text {trap }}[11]$. This shift is illustrated in Fig. 2.

Thus we can investigate trapping as follows: Fix the wiggler and beam parameters, and use a wiggler length shorter than the length required to produce synchrotron oscillations at all RF input powers. Slowly increase the RF input power $P_{\text {in }}$ while continually monitoring the beam energy that gives the maximum gain, $\gamma_{\text {peak }}$. When the input power $P_{\text {in }}$ is low, $\gamma_{\text {peak }}=\gamma_{\text {plin }}$. Eventually the input power will be sufficient to cause the FEL interaction to be nonlinear-thereafter $\gamma_{\text {peak }}$ will steadily increase. By determining the energy increase curve characteristics as a function of various system parameters, we can map out the FEL ponderomotive trapping bucket. Note that the above simplistic arguments are not entirely born out by proper theoretical[16] and computation analysis, but are nevertheless a very useful guide.

We use the MIT X-Band collective free electron laser. This device has previously been extensively described and characterized $[9,12,17]$. In brief, the FEL uses a thermionic cathode electron gun to produce a low emittance, $\gamma \approx 1.3, r=0.254 \mathrm{~cm}$, several ampere electron beam. Decreasing the cathode heater current forces the gun into the temperature limited regime, and thus can be used to decrease the beam current. The beam power ranges up to approximately $210 \mathrm{~kW}$. The input microwave power $(f=9.31 \mathrm{GHz}, P<$ $15 \mathrm{~kW})$ is superimposed on the electron beam by a coupler, and the beam and microwaves then stream into a $l=3.5 \mathrm{~cm}$, bifilar helical wiggler. The beam is guided by a $1580 \mathrm{G}$ axial magnetic field. The beam and microwaves propagate in standard WR-90 X-Band stainless steel waveguide in the lowest order mode $\left(\mathrm{TE}_{10}\right)$, and the beam space charge reduction factor is $p_{1} \approx 0.5[18]$. The beam is dumped by a kicker magnet at $0.7 \mathrm{~m}$. The gain is generally less than two. The output power is detected by a standard directional coupler, variable attenuator, crystal detector setup. The system is powered by a Physics International 615MR Marx generator; a crucial feature of which is that its output voltage pulse closely resembles that of a decaying $\mathrm{RC}$ circuit. Thus we get an entire voltage/energy scan on every shot.

In Fig. 3 we show the peak gain energy $\gamma_{\text {peak }}$ as a function of input power $P_{\text {in }}$ for five values of the wiggler field. The beam current is held constant 
at $I=0.6 \mathrm{~A}$. The experimental data clearly shows an increase in $\gamma_{\text {peak }}$ as the input power is increased, and is in good agreement with the computer simulations. The nonlinear onset occurs at lower input power as the wiggler field increases. Experimental fluctuations interfere with assigning an exact "knee" point to each curve. Indeed, the computer simulations indicates that the knee has a soft onset. However if a somewhat arbitrarily identified knee point is assigned to each curve (at the RF input power $P_{\text {in }}$ at which the beam energy $\gamma_{\text {peak }}$ is statistically significantly greater than the linear gain beam energy $\left.\gamma_{\text {plin }}\right)$, the relation $B_{w} P_{\text {knee }}^{1 / 2}$ is loosely invariant[19] as predicted by Eq. 3. Similar curves for the energy that gives the maximum decrease in output power (the fast wave interaction) were also found (See also Fig. 2). We found (not shown) that the dip knee is at a much higher RF power than the gain knee. This is readily understood by remembering that the fast wave interaction absorbs RF energy, consequently, for otherwise identical parameters, the RF power available for trapping is lower.

In Fig. 4 we show the peak gain energy $\gamma_{\text {peak }}$ as a function of input power $P_{\text {in }}$ for 4 values of the beam current $I$. The wiggler field was fixed at $B_{w}=238 \mathrm{G}$. Since the plasma frequency scales with the beam current, we would expect the required knee RF power to increase with the beam current. More precisely, Eq. 2 demonstrates that larger plasma frequencies lead to larger linear peak gain energies $\gamma_{\mathrm{plin}}$, and thus the onset of nonlinear effects seems to require that the bucket height to be higher. Scaling of Eq. 3 indicates that $P_{\text {knee }} \propto \omega_{p}^{4} \propto I^{2}$. Experimentally, we observed an effect in the appropriate direction with approximately the appropriate magnitude. However the magnitude of the effect found in simulations of the beam current on $P_{\text {knee }}$ is significantly smaller, though in the appropriate direction. We do not at present understand this discrepancy. Further experimental and theoretical study is necessary to resolve this question.

The comparison between experiment and simulation requires accurate knowledge of the plasma frequency $\omega_{p}$, corrected for relativistic, finite radius, axial field and other effects (See Eq. 1). Because the beam plasma frequency is the more fundamental quantity, we employ a direct experimental measure of the corrected $\omega_{p}$, rather than a measurement of the beam current $I$ and subsequent calculation of $\omega_{p}$. Consequently we avoid many classes of experimental error. To determine the plasma frequency directly we measure the energy split between the linear fast and slow wave interactions; 
i.e. between the gain peak and dip in the linear power output versus beam energy curve (see Eq. 1 and Fig. 2). This method has previously been shown to be an accurate measure of the beam current[12], and the beam currents reported in Figs. 3 and 4 are those inferred from the corrected $\omega_{p}$.

The computer simulation employed in these figures is explained in detail elsewhere[11]. The code utilizes an extended one-dimensional model to track the nonlinear electron motions in the $(\gamma, \Psi)$ space. Instead of assuming a helical wiggler field with constant amplitude, the code includes Bessel functions in the wiggler field to generate more realistic electron orbits[20]. The presence of an axial magnetic field and $\mathrm{rf}$ space charge are also included. The signal power is then given by the self-consistent coupling between the electron beam and the fundamental $\mathrm{TE}_{10}$ mode.

Many possibilities have been considered in trying to reconcile the differencies in Fig. 4 between experiment and simulation. We have considered errors in the simulation itself such as inadequate time steps or particle numbers, as well as physical phenomena such as varying beam temperatures. Unfortunately no explanation was found. The discrepancy may be due to the inadequacy of the simplified model used in the code, namely excluding the three-dimensional effects, the changes in the effective beam radius with changes in $B_{w}$ and $I$ and the non-ideal electron orbits induced by the large RF signal.

In conclusion, we have found a new nonlinear effect in free electron lasers. The effect is directly related to ponderomotive bucket trapping, the fundamental FEL saturation mechanism. Thus we have found a new "probe" to study saturation. The high input power regime we have investigated constitutes a new method to study, with low energy beams, the mode in which low gain saturated oscillator FELs operate.

We gratefully acknowledge the comments and support of G. Bekefi. This work was funded by the National Science Foundation, the Office of Naval Research, the U.S. Department of Energy, Division of Nuclear and High Energy Physics, and by internal U.C. Berkeley funds. Some of this work was performed at UCSD. 


\section{References}

[1] D. A. G. Deacon, L. R. Elias, J. M. J. Madey, G. J. Ramian, H. A. Schwettman, and T. I. Smith, "First Operation of a Free-Electron Laser", Phys. Rev. Lett. 38, p892-894 (1977).

[2] M. Billardon, P. Elleaume, J. M. Ortega, C. Bazin, M. Bergher, M. Velghe, D. A. G. Deacon, and Y. Petroff, "Free-Electron Laser Experiments at Orsay: A Review" IEEE J. Quantum Electron. QE-21, p805-823 (1985).

[3] J. Mathew and J. A. Pasour, "High Gain, Long-Pulse Free-Electron Laser Oscillator", Phys. Rev. Lett. 56, p1805-1808 (1986).

[4] J. Masud, T. C. Marshall, S. P. Schlesinger, and F. G. Yee, "Gain Measurements From Start-Up and Spectrum of a Raman Free-Electron Laser Oscillator", Phys. Rev. Lett. 56, p1567-70 (1986).

[5] D. W. Feldman, R. W. Warren, B. E. Carlsten, W. E. Stein, A. H. Lumpkin, S. C. Bender, G. Spalek, J. M. Watson, L. M. Young, J. S. Fraser, J. C. Goldstein, H. Takeda, T. S. Wang, K. C. D. Chan, B. D. McVey, B. E. Newnam, R. A. Lohsen, R. B. Feldman, R. K. Cooper, W. J. Johnson, and C. A. Brau, "Recent Results from the Los Alamos Free-Electron Laser", IEEE J. Quantum Electron. QE-23, p1476-1488 (1987).

[6] T. J. Orzechowski, B. R. Anderson, W. M. Fawley, D. Prosnitz, E. T. Scharlemann, S. M. Yarema, D. B. Hopkins, A. C. Paul, A. M. Sessler, and J. W. Wurtele, "Microwave Radiation from a High Gain Free-Electron Laser Amplifier", Phys. Rev. Lett. 54, p889 (1985).

[7] T. J. Orzechowski, B. R. Anderson, J. C. Clark, W. M. Fawley, A. C. Paul, D. Prosnitz, E. T. Scharlemann, S. M. Yarema, D. B. Hopkins, A. M. Sessler, and J. W. Wurtele, "High-Efficiency Extraction of Microwave Radiation from a Tapared-Wiggler Free-Electron Laser", Phys. Rev. Lett. 57, p2172-2175 (1986).

[8] S. H. Gold, W. M. Black, H. P. Freund, V. L. Granatstein, and A. K. Kinkead, "Radiation Growth in a Millimeter-Wave Free-Electron Laser Operating in the Collective Regime", Phys. Fluids 27, p746-754 (1984). 
[9] J. Fajans, J. S. Wurtele, G. Bekefi, D. S. Knowles, and K. Xu, "Nonlinear Power Saturation and Phase (Wave Refractive Index) in a Collective Free-Electron Laser amplifier", Phys. Rev. Lett. 57, p579-582 (1986).

[10] R. W. Warren, B. E. Newnam, J. G. Winston, W. S. Stein, L. M. Young, and C. A. Brau, "Results of the Los Alamos Free-Electron Laser Experiment", IEEE J. Quantum Electron. QE-19, p391-401 (1983).

[11] J. S. Wurtele, R. Chu, and J. Fajans, "Nonlinear Theory and Experiment of Collective Free-Electron Lasers", Phys. Fluids B 2, p1626-1634 (1990).

[12] J. Fajans, G. Bekefi, Y. Z. Yin, and B. Lax, Phys. Fluids "Microwave Studies of Tunable Free-Electron Laser in Combined Axial and Wiggler Magnetic Fields", 28, p1995-2006 (1985).

[13] H. P. Freund and P. Sprangle, "Unstable Electrostatic Beam Modes in Free-Electron Laser Systems", Phys. Rev. A 28, p1835-1837 (1983).

[14] N. M. Kroll, P. L. Morton, and M. N. Rosenbluth, "Free-Electron Lasers with Variable Parameter Wigglers", IEEE J. Quantum Electron. QE17, p1436-1468 (1981).

[15] Space charge effects do not significantly modify this formula when the beam is unbunched. However, when the beam is bunched the space charge forces will significantly perturb the trapping buckets[11].

[16] J. Fajans, J.S. Wurtele and R. Chu, "Parametric Studies of the FEL Bucket Height", Bull. Am. Phys. Soc. 34 p1986 (1989) and R. Chu, unpublished.

[17] J. Fajans and G. Bekefi, "Measurements of Amplification and Phase Shift (Wave Refractive Index) in a Free-Electron Laser", Phys. Fluids 29, p3461-3469 (1986).

[18] G. M. Branch and T. G. Mihran, "Plasma Frequency Reduction Factors in Electron Beams", IRE Trans. Elec. Dev. ED-2, p3-11 (1955).

[19] However, the best fit to the data does not yield this relation exactly. 
[20] J. Fajans, D. A. Kirkpatrick, and G. Bekefi, "Off-Axis Electron Orbits in Realistic Helical Wigglers for Free-Electron Laser Applications",Phys. Rev. A, 32, p3448-3453 (1985). 
Fig. 1. FEL phase space $(\Psi, \gamma)$ showing the ponderomotive trapping buckets, the maximum gain energy, the resonant energy, and the maximum absorption energy. The larger bucket corresponds to larger input power.

Fig. 2. Detuning curves showing gain vs. beam energy in the linear regime (solid line, $P_{\mathrm{in}}=15 \mathrm{~W}$ ) and the nonlinear (dashed line, $P_{\mathrm{in}}=15 \mathrm{~kW}$ )regime. In both cases $B_{w}=184 \mathrm{G}$ and $I=0.6 \mathrm{~A}$.

Fig. 3 Energy of peak gain $\gamma_{\text {peak }}$ vs. $P_{\text {in }}$ for five values of the wiggler magnetic field. Note the progression to lower RF powers at higher wiggler fields for the nonlinear onsets. The dots are experimental data, and the lines are computer simulations. Note that the simulations is not extended into the region of highest wiggler field and input power because the electrons approach gyro-resonance in this region and the simulation analytic orbit approximations break down.

Fig. 4 Energy of peak gain $\gamma_{\text {peak }}$ vs. $P_{\text {in }}$ for 4 values of the beam current. The dots are experimental data. The lines are computer simulations. (a) $I=$ $1.35 \mathrm{~A},(\mathrm{~b}) I=0.9 \mathrm{~A}$, (c) $I=0.6 \mathrm{~A}$, and (d) $I=0.2 \mathrm{~A}$. 


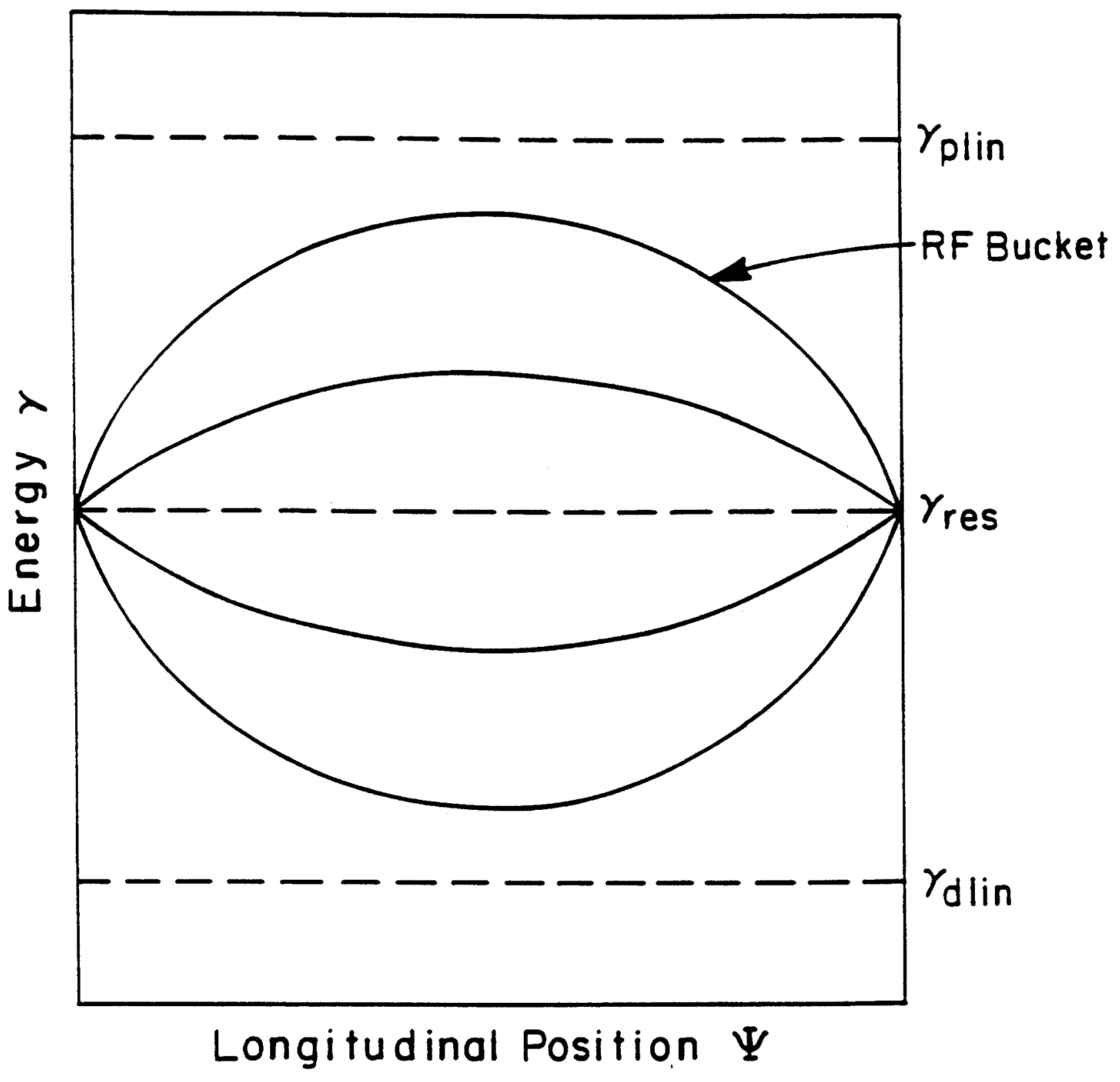




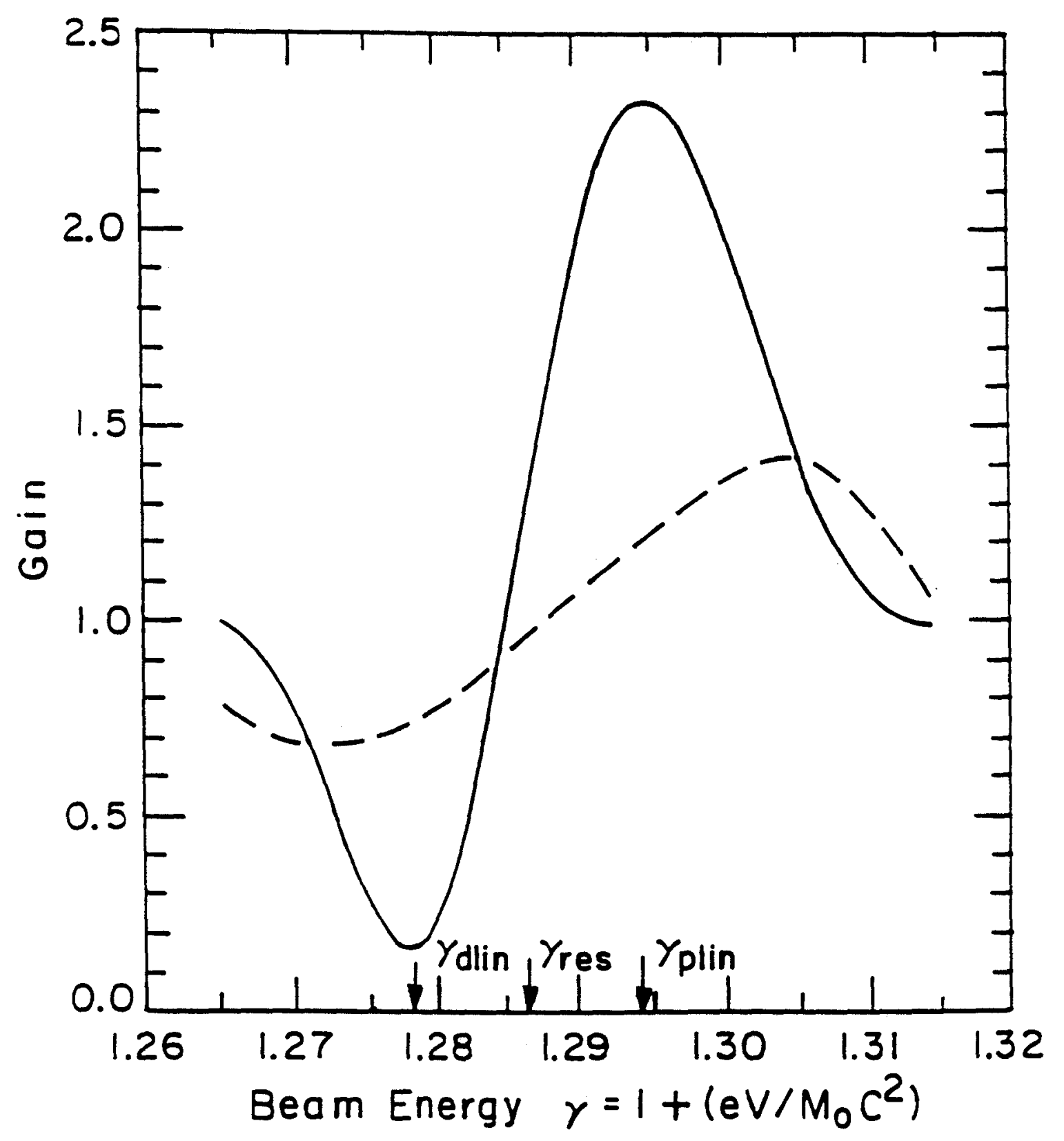

Fig. 2 Fajans, Chu, Wurtele 


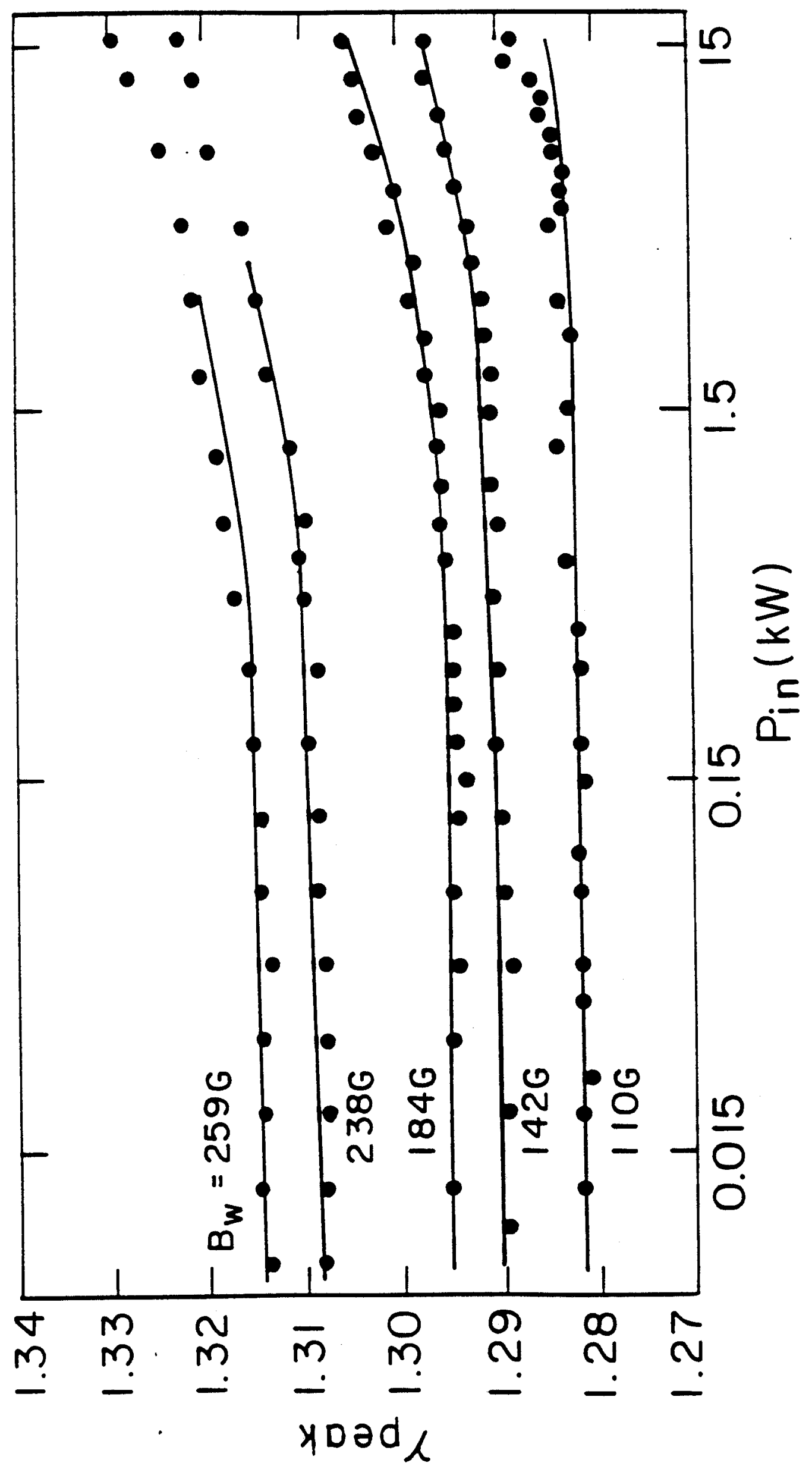




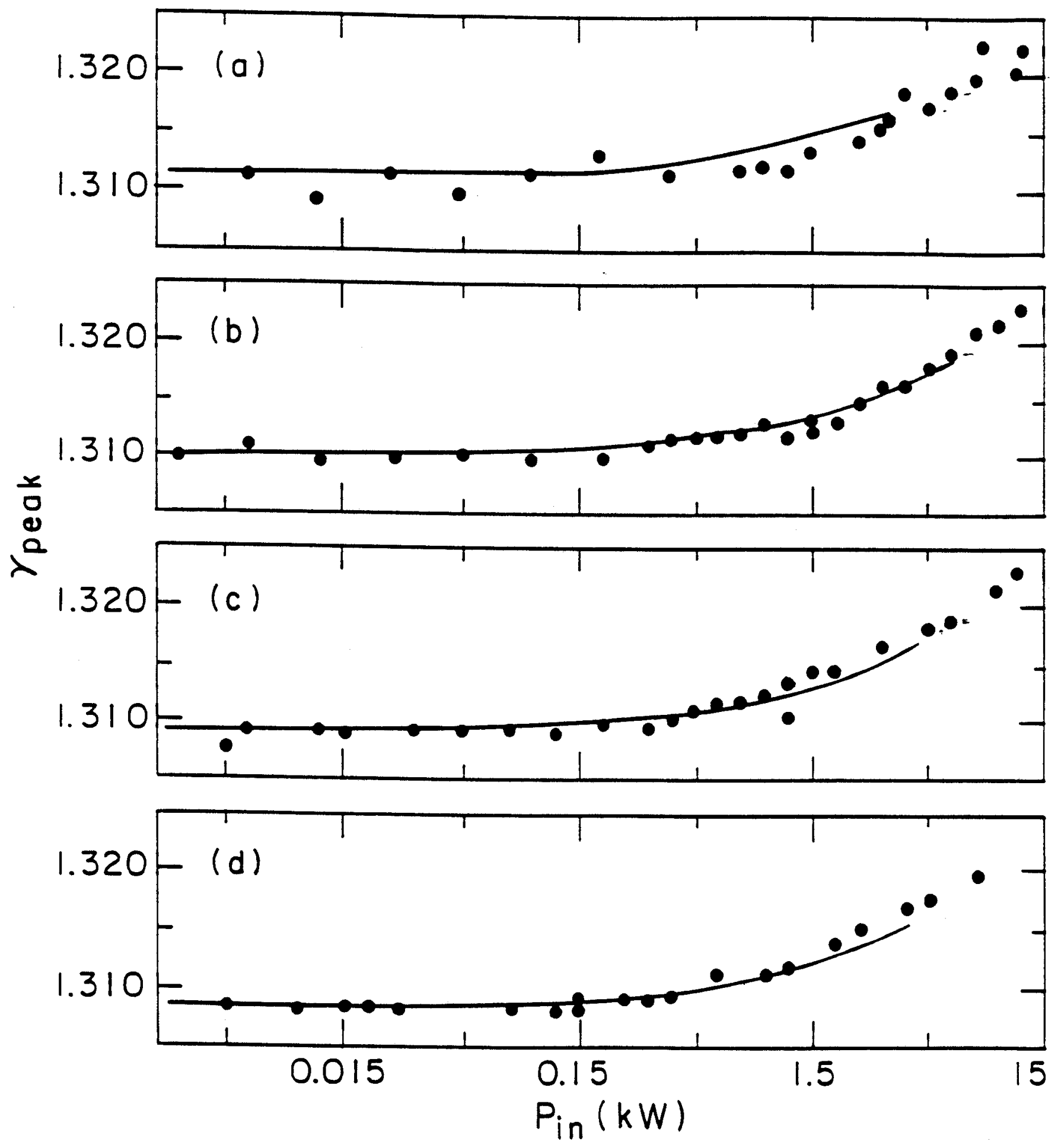

Fig. 4 Fajans, Chu, Wurtele 